\title{
Correction to: Performance Evaluation of a Newly Developed MR-Compatible Mobile PET Scanner with Two Detector Layouts
}

Masao Watanabe, ${ }^{1}$ Yuji Nakamoto, ${ }^{1}$ Ryusuke Nakamoto, ${ }^{2}$ Takayoshi Ishimori, ${ }^{1}$ Tsuneo Saga, ${ }^{1}$ Kaori Togashi ${ }^{1}$

${ }^{1}$ Department of Diagnostic Imaging and Nuclear Medicine, Graduate School of Medicine, Kyoto University, 54 Shogoinkawahara-cho, Sakyo-Ku, Kyoto, 606-8507, Japan

${ }^{2}$ Department of Radiology, Shiga General Hospital, 5-4-30 Moriyama, Moriyama, Shiga, 524-8524, Japan

Correction to: Mol Imaging Biol

https://doi.org/10.1007/s11307-019-01384-9

This article was updated to correct the "greater than or equal to" $(\geq)$ symbols in Tables 4 and 5, which incorrectly appeared as "greater than" $(>)$ symbols.

Publisher's Note Springer Nature remains neutral with regard to jurisdictional claims in published maps and institutional affiliations.

The online version of the original article can be found at https://doi.org/ 10.1007/s11307-019-01384-9

Correspondence to: Yuji Nakamoto; e-mail: ynakamo1@kuhp.kyoto-u.ac.jp 